\title{
DIFERENCIA, AUTODEFINICIÓN Y EMPODERAMIENTO: UNA LECTURA NECESARIA DE SISTER OUTSIDER DE AUDRE LORDE
}

\author{
Difference, self-definition and Empowerment: \\ a necessary interpretation of Sister Outsider by Audre Lorde
}

\author{
María Laura Cucinotta* \\ Universidad de Buenos Aires \\ marialauracucinotta@gmail.com
}

Palabras clave

diferencia;

autodefinición;

empoderamiento;

interseccionalidad

Keywords

difference;

self-definition;

empowerment;

intersectionality

\section{Resumen}

En este artículo breve me propongo describir y analizar las formas de resistencia al sexismo y la homofobia que postula Audre Lorde en algunos en los ensayos y conferencias reunidos en Sister Outsider (1984). Asimismo, pretendo demostrar que dichos textos pueden entenderse como intervenciones políticas sistemáticas que tienen como objetivo principal construir espacios de expresión donde confluyan las voces de actores sociales históricamente relegados. En el marco de su trabajo en prosa, Lorde abordó y exploró temáticas tales como el orgullo, el amor, la violencia, el temor racial, la opresión sexual, los modos de vida urbanos y las diversas formas de supervivencia en la gran ciudad. Según la base teórica de los estudios de género y con el foco puesto específicamente en las producciones teóricas del feminismo afroestadounidense, pretendo demostrar la importancia del aporte de Lorde a la construcción del concepto de interseccionalidad, así como también a la visibilización de múltiples situaciones de violencia sucedidas al interior de la comunidad negra.

\begin{abstract}
In this short article I will describe and analyze the forms of resistance towards sexism and homophobia that Audre Lorde postulates in some of the essays and speeches gathered in Sister Outsider (1984). Additionally, I would like to demonstrate that those texts can be understood as systematical political interventions that look forward the construction of spaces of expression where all the voices of historically relegated subjects can converge. In the context of her prose writing, Lorde addressed and explored diverse concepts such as pride, love, violence, racial fear, sexual oppression, ways of urban living and the different forms of surviving in the big city. Starting with the theoretical base of Black Feminism, I pretend to prove the importance of Lorde's contribution to the concept of intersectionality as well as the visibilization of multiple situations of violence inside the black community.
\end{abstract}




\section{Diferencia, autodefinición y empoderamiento: una lectura necesaria de Sister Outsider de Audre Lorde}

El pensamiento occidental europeo concibe el mundo en términos de oposición. Audre Lorde sostiene que para que dicho condicionamiento sea efectivo y se mantenga estable a lo largo de la historia, es necesario que exista un grupo que ocupe el espacio desagradable y deshumanizado de lo inferior, lo bajo, lo incivilizado, y otro grupo que, por el contrario, considere inherente su derecho a ser superior y a dominar. Para sobrevivir, los miembros del primer grupo -los afrodescendientes, los pueblos no europeos, las mujeres, los viejos, la clase obrera- deben no solo soportar el peso de la opresión sino también deben emplear sus energías en la asimilación del lenguaje y las costumbres de la clase dominante. Preocupada por lo que ella misma consideraba una tendencia moderna a la categorización de los seres humanos en grupos, Lorde dedicó su vida y su talento creativo a la lucha contra las injusticias del racismo, el sexismo y la homofobia en una sociedad fuertemente marcada por la violencia y la discriminación.

A lo largo de este artículo breve, me propongo analizar algunos conceptos centrales del pensamiento de Audre Lorde, muchos de los cuales se desprenden de su concepción sobre las diferencias. Crítica de toda política que promoviera el separatismo entre hombres y mujeres negras pero también entre feministas blancas y negras, Lorde entendió la importancia de tejer alianzas superadoras de las diferencias que fueran más allá de la comunidad de pertenencia. ${ }^{1}$ Para ella las diferencias no deben observarse con tolerancia sino analizarse y entenderse como un reservorio de polaridades a partir de las cuales nacerá la creatividad. Pero eso solo podrá darse en un marco de interdependencia de las diversas fuerzas que, reconocidas en un plano de igualdad, busquen generar nuevas formas de ser y de estar en el mundo.

Promover la tolerancia de las diferencias entre mujeres implica para Lorde negar la función creativa que estas tienen. Por su parte, todas las formas de odio basadas en un sentimiento de superioridad son "formas de la ceguera humana". En Scratching the Surface: some notes on barriers to women and loving (1978) las enumera y define:

Racismo: creencia en la superioridad inherente de una raza sobre el resto y su consiguiente derecho a dominar.

Sexismo: creencia en la superioridad inherente de un sexo y su consiguiente derecho a dominar.

Heterosexismo: creencia en la superioridad inherente de una forma de amar y su consiguiente derecho a dominar.

1. Un ejemplo es la relación que cultivó con la poeta, feminista y activista lesbiana blanca Adrienne Rich, una amistad cultivada a partir de las diferencias, que potencia el pensamiento crítico y la creatividad. 
Homofobia: temor a experimentar sentimientos amorosos hacia miembros del mismo sexo y su consiguiente rechazo a esos sentimientos en los otros. ${ }^{2}$ (Lorde, 2007, p. 45) ${ }^{3}$

Esos tipos de discriminación responden a una inhabilidad para reconocer "las diferencias" como una fuerza humana dinámica, útil y enriquecedora. Lorde ha sido una ferviente defensora de las diferencias para el desarrollo de un movimiento feminista que considere a las mujeres negras y sus experiencias en el mundo real. Asimismo, se encargó de denunciar a quienes, desde lugares de poder, ignoran las diferencias y se aprovechan de las mismas para construir espacios de privilegio. Para Lorde las diferencias pueden ignorarse, tratar de imitarse, cuando se consideran superiores, o buscar eliminarse, cuando se las percibe como inferiores. En ese contexto lo que es diferente se considera inferior pero también como amenazador.

Las implicancias del sexismo y de la homofobia, por ejemplo, pueden comenzar a entenderse si se visualiza cómo las diferencias han servido para justificar todo tipo de acto de discriminación a lo largo de la historia de la humanidad. Pero la invisibilización histórica de estas diferencias ha llevado también al silencio sobre situaciones de desigualdad al interior de movimientos sociales tales como el feminismo o los grupos que reclamaban derechos sociales para las minorías en los Estados Unidos.

La apreciación positiva del concepto de diferencia que hace la autora abre un abanico de pluralidades y posibilidades diversas de ser y habitar el mundo a la vez que sienta las bases para la lucha en contra de cualquier tipo de opresión (sexista, racista, patriarcal). Asimismo, se trata de un acto que favorece el desarrollo de relaciones interpersonales más ricas en las que se considera a las personas en sus complejidades. Finalmente, las reflexiones de Lorde en torno al concepto de diferencia terminaron siendo un aporte fundamental y decisivo al movimiento feminista.

Si bien es cierto que sin comunidad no hay liberación posible, su existencia no debe suponer una pérdida de las diferencias de sus miembros, ni la pretensión de que dichas diferencias no existan. Para Lorde las mujeres que se mantienen firmes fuera del círculo de lo que la sociedad define como mujeres aceptables saben que la supervivencia no es una asignatura que se aprende en las universidades. Las mujeres negras tienen que aprender a resistir y hacer causa común con otras que también están fuera del sistema. A fin de cuentas, la supervivencia implica aprender a asimilar las diferencias y a convertirlas en potencialidades. En una economía basada en la acumulación de capital, que considera outsiders a todos aquellos que no se apegan a la norma, rechazar las diferencias se convierte en una actividad necesaria. En este contexto, Lorde postula tres formas de actuar frente a las diferencias: se las puede ignorar, utilizarlas para imitar-si creemos que esa diferencia marca un rango de superioridad con respecto a mi persona- o destruirlas -cuando se considera que el diferente es un subordinado. En Age, Race, Class and Sex: women redefining difference (1980) Lorde llama a reconocer las diferencias y

\footnotetext{
2. "Racism: The belief in the inherent superiority of one race over all others and thereby the right to dominance. Sexism: The belief in the inherent superiority of one sex and thereby the right to dominance.

Heterosexism: The belief in the inherent superiority of one pattern of loving and thereby its right to dominance. Homophobia: The fear of feelings of love for members of one's own sex and therefore the hatred of those feelings in others".

3. Todas las traducciones de citas en este artículo me pertenecen.
} 
analizar los efectos que tienen sobre el comportamiento humano. Además, agrega que ignorar las diferencias entre mujeres constituye una amenaza seria al poder articulado de las afroestadounidenses que, a diferencia de las blancas, tienen que poder separar las necesidades del opresor de sus propios conflictos dentro de la comunidad y entender que la opresión racista opera de diferentes maneras sobre los hombres y las mujeres negras, así como la opresión patriarcal subyuga utilizando diferentes métodos según el color de la mujer. Kelly Lynch Reames señala que las diferencias a las que hace referencia Lorde en su trabajo deben entenderse como formas de poder y no como fuerzas divisorias: “aceptar los límites definitivos de cada categoría -raza, género, clase, etcétera- mientras se ocultan sus limitaciones, implica una profundidad y una complejidad en la construcción de la identidad que refuta toda una historia de limitaciones"4 (1994, p. 696). Suprimir la raza como categoría de análisis es un problema para las mujeres blancas y para las negras porque de esta forma se replica la invisibilidad que refuerza la división entre mujeres.

El constante enfrentamiento de Lorde con notables figuras del movimiento feminista la condujo a convertirse en una suerte de outsider, una voz aislada que acusaba con furia a las propias feministas de reproducir modelos patriarcales al interior del colectivo de mujeres. Según la autora, el feminismo más ortodoxo no consideraba diferencias en la categoría de mujer y tendía a generalizar la experiencia femenina. Los sujetos que se encuentran por fuera de lo que Lorde denominó la "norma mítica" -es decir, quienes no son blancos, delgados, hombres, jóvenes, heterosexuales, cristianos y financieramente solventes- suelen identificar una forma en la que son diferentes y asumen que esa diferencia es la causa principal de toda opresión. Según Lorde, eso es lo que se da entre las mujeres blancas que se centran en su opresión por ser mujeres e ignoran las diferencias de raza, preferencia sexual, clase y edad. Del otro lado del debate, muchas feministas blancas acusaban a Lorde de querer asumir una identidad y una autoridad moral basadas en el sufrimiento, sentimiento que debía pensarse como una experiencia universal para las mujeres, tuvieran el color que tuviesen, y no aplicable solamente a las mujeres negras. Lynch Reames sostiene que, mediante su trabajo crítico, Lorde exige que el movimiento feminista termine con su exclusividad e incluya a las mujeres pobres y de color, además de reconocer las diferencias cruciales que existen entre las experiencias de opresión de las mujeres blancas de clase media que se consideraron predominantes hasta el surgimiento de la Segunda Ola del feminismo: "Para Lorde, la unidad y la comunidad política no implican ignorar las diferencias; en todo caso, entiende el reconocimiento de las diferencias como necesario para la comunidad" (Lynch Reames, 2007, p. 82).

Lorde utiliza un motivo recurrente en su prosa y en su poesía: la maternidad. En Man Child: $A$ Black Lesbian Femnist's Response (1979) reflexiona sobre la crianza de su hijo varón y negro en el contexto de una familia compuesta por un niño, una niña y dos madres afrodescendientes y feministas, hacia fines de la década de 1970 en los Estados Unidos. En este artículo su propuesta es poner en primer plano el reconocimiento de las particularidades de una estructura que programa a los varones

4. "embracing the definitive boundaries of each category -race, gender, class, etcetera- while dissembling their static limitations, assumes a depth and complexity of identity construction that refutes a history of limitation".

5. "For Lorde, political unity and community does not mean ignoring difference; rather, she views recognizing difference as necessary for community". 
para despreciar a las mujeres tanto como a su propia negritud. Asimismo, expresa su deseo de criar un hijo negro que no sea destruido ni corrompido por el poder blanco heterosexual; un hombre que reconozca que su opresión proviene de la particular estructura de un programa que lo prepara para odiar a las mujeres y odiarse a sí mismo. En el sistema patriarcal que rige las relaciones sociales Lorde piensa que la maternidad es el único poder abierto para todas las mujeres por el carácter transformador que tiene una crianza responsable. La experiencia de la maternidad tiene un peso muy fuerte a la hora de considerar las diferencias entre mujeres negras y mujeres blancas. Para Lorde las mujeres negras y sus hijos saben que la tela de sus vidas está cosida con violencia y con odio y que existen ciertos problemas que las mujeres blancas y negras comparten y otros que no:

Ustedes temen que sus hijos crezcan para unirse al patriarcado y testifiquen en contra suyo, nosotras tememos que arranquen a nuestros hijos de un auto y les disparen en la calle, y que ustedes les den la espalda a los motivos por los que ellos murieron. ${ }^{6}$ (Lorde, 2007, p. 119)

Según Lorde no son las diferencias per se las que separan a las mujeres sino la negativa o reticencia a reconocer esas diferencias y manejar las distorsiones que resultan de ignorarlas o silenciarlas. En ese sentido, los aportes de Lorde se vuelven fundamentales para la elaboración de una teoría de la interseccionalidad ya que la ausencia de consideraciones sobre las diferencias de raza, sexualidad, clase social y edad, debilitan cualquier discusión feminista sobre lo personal y lo político. La propuesta de la autora parte de un proceso de erradicación consciente de los patrones de opresión que todos los individuos llevan internalizados, con el objetivo de avanzar hacia un trabajo conjunto en pos del cambio social. Pero para lograrlo es necesario ser capaces de reconocer las diferencias que existen entre mujeres y utilizarlas para enriquecer todas las perspectivas. Ese es el único camino que Lorde ve como posible para lograr la unión en la lucha feminista.

Cheryl Higashida retoma el discurso que Lorde pronunció en 1982 como parte de la celebración por el Malcolm X Weekend (llevado adelante en la Universidad de Harvard) con el objetivo de analizar el concepto de diferencia que propone la autora. Para Higashida, Learning from the 60s examina las posibilidades políticas que fueron más allá del recorrido realizado por el Black Power. Al retomar la figura de Malcolm X, Lorde revisa el legado del líder político y realiza dos operaciones: por un lado, señala el trato que la Nación del Islam le dio a las mujeres, pero por el otro, destaca que, en su último año de vida, Malcolm X supo reconocer la noción de diferencia como una fuerza creativa y necesaria para generar el cambio social a través de un análisis de las condiciones bajo las cuales debían darse las alianzas y coaliciones:

Aunque reconoce la "energía brutal de la determinación negra lanzada en los 60s", que "todavía se siente en los movimientos por el cambio entre mujeres, otras personas de color, gays [y] discapacitados", Lorde insiste en que sus lectores confronten y trasciendan las limitaciones del

6. "You fear your children will grow up to join the patriarchy and testify against you, we fear our children will be dragged from a car and shot down in the street, and you will turn you backs upon the reasons they are dying". 
nacionalismo: "debemos enfrentar con claridad y perspicacia las lecciones que aprendimos de la simplificación excesiva de cualquier lucha por la autoconciencia y la liberación, o no reuniremos la fuerza que necesitamos para enfrentar las amenazas multidimensionales que desafían nuestra supervivencia en los 80s.” Todo eso convive con los reclamos postnacionalistas que la segunda ola del feminismo negro, de la cual este ensayo es un ejemplo, expone al romper con las políticas esencialistas y regulatorias del nacionalismo negro. ${ }^{7}$ (Higashida, 2013, p. 140)

Lorde supone que, de seguir vivo, Malcolm X hubiera orientado su militancia al trabajo sobre las diferencias y a demostrar que no deben provocar temor sino considerarse una fuerza creativa. La sociabilización y aceptación de las diferencias supone una instancia de aprendizaje y crecimiento conjunto. El legado de Malcolm que rescata Lorde tiene que ver con su examen sobre las condiciones sociales bajo las cuales deben realizarse alianzas y coaliciones orientadas al cambio social.

Para Higashida, las diferencias sexuales y raciales que señala Lorde no podrían prosperar en un orden que no fuera neoliberal ni neocolonial. Es por eso que las batallas por la autodeterminación, el reconocimiento de la cultura nacional propia y el reclamo por las tierras fueron las bases principales para la validación de la interseccionalidad y la heterogeneidad. Lorde conceptualiza una relación dialógica oposicional entre la liberación nacional y el internacionalismo. De la misma manera, se planteaba en las agendas de las mujeres afroalemanas que conoció mientras enseñaba poesía en Berlín, y lo mismo puede decirse de las líderes de los movimientos indígenas antipatriarcales y otras escritoras negras.

Estelle Freedman (2006) sostiene que durante las décadas de 1970 y 1980 se generaron diversas coaliciones políticas que desafiaron al feminismo de los Estados Unidos. Según su relato, hubo grupos independientes de mujeres negras que notaron que dentro de sus comunidades los temas que representaban un problema mayor tenían que ver con el acceso al sistema de salud y los beneficios sociales otorgados por el gobierno. Según Freedman, esas mujeres no abandonaron la causa feminista, sino que tomaron las diferencias que las apartaban de sus compañeras de lucha blancas y las transformaron en fortalezas. Una de esas primeras transformaciones es la que Lorde denomina "La transformación del silencio en lenguaje y acción” (2007, p. 40-44). En el ensayo que lleva el mismo título la autora comienza haciendo una referencia a su propia vida y cuenta que el primer tumor maligno que le detectaron la condujo a tomar conciencia de su mortalidad, poner su vida en perspectiva y profundizar aspectos básicos de su existencia: "Me he convencido de que es necesario expresar, verbalizar, compartir aquello que es importante para mí, aun a riesgo de que se me

7. 'While acknowledging the 'raw energy of black determination released in the 60s', which 'is still being felt in movements for change among women, other peoples of Color, gays [and] the handicapped', Lorde urges her readers to confront and transcend the limitations of nationalism: 'We must face with clarity and insight the lessons to be learned from the oversimplication of any struggle for self-awareness and liberation, or we will not rally the force we need to face the multidimensional threads to our survival in the 80s.' All of this is keeping with postnationalist claims that second-wave Black feminism, of which this essay is an example, exposes and breaks with essentialist and regulatory black nationalist politics". 
malinterprete o tergiverse. Creo que, por encima de todo, hablar me beneficia” (Lorde, 2007, p. 40). Lorde entiende que el silencio es sinónimo de muerte y que la muerte es para ella el único silencio final. La fortaleza a la que se refiere en su ensayo radica en poner el miedo en perspectiva y asumir la necesidad de desatar una guerra contra la tiranía del silencio.

En los Estados Unidos, donde la diferencia racial crea una distorsión constante de la visión, las mujeres negras han sido, por un lado, siempre muy visibles y por el otro, invisibilizadas a partir de la despersonalización del racismo. En otras palabras, las mujeres negras son especialmente visibles y propensas a la objetivación. Paradójicamente, ese mismo tratamiento las convierte en potencialmente invisibles como seres humanos. Hill Collins (2002) retoma esa afirmación y explica que, para Lorde, el doble juego de la visión al que están sometidas las mujeres negras tiene que convertirse en una fuente de poder, basada en la autodeterminación:

Para sobrevivir en las fauces de este dragón que llamamos Estados Unidos la lección más importante y vital que tuvimos que aprender es que nunca se pretendió que sobreviviéramos. No como seres humanos. La maquinaría tratará de triturarlas en cualquier caso, tanto si hablan como si callan. Podemos permanecer eternamente mudas en un rincón mientras nuestras hermanas y nosotras mismas nos consumimos, mientras se deforma y destruye a nuestros hijos, mientras se envenena nuestra tierra; podemos sentarnos en nuestros cómodos rincones, mudas como botellas, y no por ello tendremos menos miedo. ${ }^{9}$ (Lorde, 2007, p. 42)

Lorde cree que la supervivencia es una destreza que no se aprende en ningún espacio de educación formal porque nadie enseña a las mujeres negras a moverse en soledad, entre el rechazo y la injuria. El deseo de sobrevivir moviliza hacia el armado de redes entre quienes se reconocen por fuera de las estructuras del mundo occidental.

"El silencio no te protegerá" es quizá una de las frases más famosas de Lorde. La intervención que propone genera miedo y rechazo, eso Lorde lo sabe, pero también sabe que el silencio encierra aún más sufrimiento. En numerosos ensayos y conferencias hace un llamado al trabajo cooperativo y en "La transformación del silencio en lenguaje y acción" lo hace a partir de la referencia a Kwanza, ${ }^{10}$ la fiesta seglar afroestadounidense que entre el 26 de diciembre y el 1 de enero celebra cada día un principio diferente. En el artículo Lorde menciona los primeros dos, ${ }^{11}$ unidad y autodeterminación, y se detiene en el tercero, trabajo y responsabilidad colectivos. La guerra contra la tiranía del silencio

8. "I have become to believe over and over again that what is most important to me must be spoken, made verbal and shared, even at the risk of having it bruised or misunderstood. That the speaking profits me, beyond any other effect". 9. "For to survive in the mouth of this dragon we call America, we have had to learn this first and most vital lesson -that we were never meant to survive. Not as human beings. (...) the machine will try to grind you into dust anyway, whether or not we speak. We can sit in our corners mute forever while our sisters and our selves are wasted, while our children are distorted and destroyed, while our earth is poisoned; we can sit in our safe corners mute as bottles, and we will still be no less afraid".

10. El término proviene del suajili “matunda ya kwanza” que significa “primeras frutas”. E1 Dr. Mulana 'Ron’ Karenga, activista negro, fundó esta fiesta en 1967.

11. Los cuatro restantes son: economía cooperativa, objetivo y dirección, creatividad y fe. 
que imagina Lorde no es un acto solitario sino un trabajo comunitario que requiere el compromiso de cada una de las partes y, en gran medida, ese compromiso es con el poder de la palabra. El proceso que describe Lorde necesita de un trabajo comunitario en el que se dividan las tareas: quienes escriben deben analizar la verdad de lo que dicen y del lenguaje con el que se comunican; quienes no escriben deben compartir y difundir las palabras que consideran importantes; todos los miembros de la comunidad, mediante la vida y la palabra, deben enseñar las verdades que conocen, aquellas en las que creen, más allá de la razón.

$\mathrm{El}$ discurso titulado The master's tools will never dismantle the master's house es central para analizar la importancia que le da Lorde a la autodefinición, proceso que considera clave a la hora de pensar el cambio social:

Porque las herramientas del amo nunca desmantelan la casa del amo. Quizá nos permitan obtener una victoria pasajera, siguiendo sus reglas, pero nunca nos permitirán lograr un cambio genuino. Y esto resulta amenazador para las mujeres que consideran que la casa del amo es su única fuente de apoyo. ${ }^{12}$ (Lorde, 2007 , p. 112)

A partir de situaciones de su vida cotidiana Lorde observa que existe una constante en la vida de las mujeres negras: la responsabilidad que tiene el oprimido de mostrar y explicar al opresor los errores que comete. De otro modo, los opresores conservan su posición y evaden la responsabilidad de sus propios actos. Las actividades que enumera Lorde constituyen un drenaje constante de energía que podría usarse en el proceso de redefinición del propio ser y en la planificación de medios realistas y posibles para modificar el presente y construir el futuro.

Para Hill Collins (2002), el discurso de Lorde está destinado a las mujeres afroestadounidenses a quienes llama a rechazar la imagen que el amo tiene de ellas y a luchar por una perspectiva de género basada en el womanism ${ }^{13}$ y la autodefinición. Según la autora, la intención de Lorde es generar una masa crítica de mujeres que busquen cambiar sus condiciones de vida y apelen a formas de poder colectivas. El feminismo forjado en la academia fracasó porque no supo reconocer las diferencias como fuerzas cruciales y poderosas y, por ende, no superó la primera lección del patriarcado: “divide y reinarás". Lorde propone superar ese error y redefinir ese principio: solo autodefiniéndose las mujeres serán capaces de empoderarse. Así es como también invita a su comunidad a realizar una revisión crítica y no idealista del pasado. La década del sesenta es sin dudas una de la más significativas si pensamos en las intervenciones de personalidades tales como Malcolm X o Martin Luther King, el surgimiento de The Black Panther Party y, posteriormente, de la Nation of Islam. En los ochenta, cuando Lorde afirma que no hay que repetir los mismos errores del pasado se refiere a evitar las luchas unidimensionales y entender la necesidad de autodefinirse; está invitando a no olvidar las lecciones

12. "For the master's tools will never dismantle the master's house. They may allow us temporarily to beat him at his own game, but they will never enable us to bring about genuine change. And this fact is only threatening to those women who still define the master's house as their only source of support".

13. El womanism es una teoría social arraigada en la opresión racista y sexual que sufren las mujeres afroestaounidenses. La escritora Alice Walker utilizó el término por primera vez en el cuento Coming apart (1979). 
del pasado, por el contrario, a interpretarlas y comprenderlas. La revolución que imagina Lorde no se dará de una vez y para siempre; por eso es necesario estar siempre al acecho de la menor oportunidad para efectuar cambios en las respuestas establecidas. Y sin lugar a duda el mejor ejemplo de la revolución que sostiene es aprender a afrontar con respeto las mutuas diferencias.

Then hearing would reconcile and he would seem to listen to two separate Quentins now-the Quentin Compson preparing for Harvard in the South, the deep South dead since 1865 and peopled with garrulous outraged baffled ghosts, listening, having to listen, to one of the ghosts which had refused to lie still even longer than most had, telling him about old ghost-times; and the Quentin Compson who was still too young to deserve yet to be a-ghost but nevertheless having to be one for all that, since he was born and bred in the deep South. (Faulkner, 1990, p. 6)

Ese sol del atardecer narra el periodo postbellum a partir del relato de Quentin como figura representativa de un periodo de crisis de estructuras del ser y el poder. En este sentido, el relato sobre Nancy -la voz de Nancy que irrumpe la narración como un rapto de emoción- no solo refleja la angustia de contacto, sino que se transforma en un catalizador de interrogantes y un desafío para la narración:

Nancy whispered something. It was oh or no, I don't know which. Like nobody had made it, like it came from nowhere and went, nowhere, until it was like Nancy was not there at all; that I had looked so hard at her eyes on the stairs that had got printed on my eyeballs, like the sun does when you have closed your eyes and there is no sun. (Faulkner, 1995, p. 296)

Estos momentos de dolorosa trascendencia suceden en la memoria de Quentin y en la de Faulkner como imágenes acústicas vívidas y difíciles de aprehender, como el sol del atardecer, como la cabeza de un fósforo ardiendo. Construcciones que se translucen a través de la relación dialéctica entre el racismo estructural del narrador, el pasado esclavista en constante reelaboración y descomposición, y una consciencia literaria que narra en vista del presente y de un público moderno, en un país unificado. Nancy es una existencia negra efímera, un recuerdo. Nancy reverbera constantemente en la repetición melódica, en imágenes discontinuadas contra toda posibilidad de narración monolítica que refleja una elaboración propia, un ensayo de respuesta a la decadencia del cuerpo y el territorio. Es, al mismo tiempo, un pecado con el que carga Quentin, un objeto sobre el que intenta infructuosamente afirmar su identidad blanca, una fuerza indomesticable exterior y una experiencia religiosa. En su no definición radica su fuerza como profunda incógnita sobre la identidad del narrador y la de su comunidad.

Algo que parece ser imposible: narrar una visión sobre la historia sureña y, a la vez, elaborar complejas metáforas, personajes inconclusos y mitos, se entrelazan armoniosamente en su literatura a través del narrador participante, cuyas características Faulkner define sobre el final de la década del veinte. Narrar lo que todavía no había sido narrado y pocos de los lectores de revistas conocían; 
recuperar lo más próximo a su historia personal y hacerlo arte elevado; crear un arte trascendente que se pregunte por la violencia de la historia: todos estos objetivos se cumplen en Ese sol del atardecer gracias a la coherencia, o la incoherencia, del narrador como partícipe de una comunidad, de sus mitos y de sus percepciones dispares y distorsionadas de la historia y la realidad. El narrador se vuelve un punto de intersección entre el realismo crítico y el arte modernista. Una muestra de un estilo capaz de captar al sur blanco en conflicto con sus culpas y sus pecados, y de dar a lo negro una presencia desafiante e indefinida para la identidad sureña. Una prosa capaz de representar la mirada directa al sol que queda impregnada en la retina aun cuando ya cerramos los ojos.

\section{Referencias bibliográficas}

Freedman, E. (2006). Feminism, Sexuality and Politics. Gender and American Culture. North Carolina, University of North Carolina.

Higashida, C. (2013). Black International Feminism. Women Writers of the Black Left 1945-1955. Illinois, University of Illinois Press.

Hill Collins, P. (2002). Black Feminist. Thought Knowledge, Consciousness, and the Politics of Empowerment. Nueva York, Routledge.

Lorde, A. (2007). Sister Outsider. New York, Crossing Press.

Lynch Reames, K. (2007). Women and Race in Contemporary U.S. Writing From Faulkner to Toni Morrison. New York, Macmillan.

* María Laura Cucinotta es Licenciada y Profesora en Letras por la FFyL, UBA. Actualmente se encuentra en el periodo de elaboración de su tesis de Maestría en Literaturas en Lenguas Extranjeras y Literaturas Comparadas que se dicta en la FFyL-UBA. Su tesis se titula Sexismo y homofobia: las formas de la resistencia según Audre Lorde y es dirigida por la Dra. Márgara Averbach. Además, se desempeña como docente en el nivel medio, superior y universitario en diversas instituciones de CABA y GBA. Finalmente, dicta charlas sobre ESI en diferentes espacios educativos y participa como investigadora asesora en el proyecto UBACYT "Hegemonía y contrahegemonía cultural en el cine histórico" dirigido por el Dr. Fabio Nigra.

RECIBIDO: 02/11/2020

ACEPTADO: 04/05/2021 